\title{
Next-to-Next-to-Leading Order Study of Three-Jet Production at the LHC
}

\author{
Michał Czakon $\odot$ \\ Institut für Theoretische Teilchenphysik und Kosmologie, RWTH Aachen University, D-52056 Aachen, Germany \\ Alexander Mitov® and Rene Poncelete \\ Cavendish Laboratory, University of Cambridge, Cambridge CB3 OHE, United Kingdom
}

(Received 25 June 2021; revised 27 August 2021; accepted 10 September 2021; published 6 October 2021)

\begin{abstract}
Multijet rates at hadron colliders provide a unique possibility for probing quantum chromodynamics (QCD), the theory of strong interactions. By comparing theory predictions with collider data, one can directly test perturbative QCD, extract fundamental parameters like the strong coupling $\alpha_{s}$, and search for physics beyond the standard model. In this work we calculate, for the first time, the next-to-next-to-leading order (NNLO) QCD corrections to typical three-jet observables and to differential three-to-two jet ratios. The calculation is complete apart from the three-jet double virtual contributions which are included in the leading-color approximation. We demonstrate that the inclusion of the NNLO corrections significantly reduces the dependence of those observables on the factorization and renormalization scales. Besides its phenomenological value, this proof-of-principle computation represents a milestone in perturbative QCD.
\end{abstract}

DOI: 10.1103/PhysRevLett.127.152001

Introduction.-The production of highly energetic sprays of particles, also known as jets, is a dominant process at hadron colliders. At high energies, where perturbation theory is expected to hold, this process offers the possibility for studying QCD in great detail. The theory-data comparison of differential multijet rates provides essential information about perturbative QCD and the modeling of jet production. The precision of these predictions is typically limited by their dependence on unphysical parameters-such as the renormalization and factorization scales-but it can be systematically increased by including higher-order corrections.

Three-jet production at the Large Hadron Collider (LHC) has been studied in great detail by experimental collaborations, see for example Refs. [1-6]. Typical observables are jet transverse momenta, angular correlations and, more generally, event-shape observables. A particular type of observable suited for perturbative QCD is the ratio $R_{3 / 2}$ of three-to-two jet rates [7]. These ratios are directly sensitive to parton splittings and are, therefore, proportional to the strong coupling constant $\alpha_{s}$. This provides an opportunity for measuring the value of $\alpha_{s}$ at the LHC. Cross section ratios have the additional advantage that some systematic uncertainties of experimental and theoretical nature cancel

Published by the American Physical Society under the terms of the Creative Commons Attribution 4.0 International license. Further distribution of this work must maintain attribution to the author(s) and the published article's title, journal citation, and DOI. Funded by SCOAP . out. A prime example is the dependence on parton distribution functions (PDFs).

There is extensive literature on theoretical predictions for multijet production through next-to-leading order (NLO) in perturbative QCD [8-13], including NLO electroweak corrections [14-16]. NLO computations have also been matched to parton showers $[17,18]$ and are generally present in multipurpose event generators [19-21]. Higher-order predictions for two-jet and single-inclusive jet production have seen extensive development in the past decade and are implemented through next-to-next-to-leading order (NNLO) in QCD [22-25]. The feasibility of NNLO QCD predictions for higher jet multiplicity is limited by the availability of two-loop virtual amplitudes and by the efficient treatment of real radiation contributions. The three-jet two-loop amplitudes have recently been made public in the leading-color approximation [26,27], leaving the real radiation as the last obstacle to predictions accurate at second order in $\alpha_{S}$.

The aim of this Letter is twofold. First, it presents NNLO QCD predictions for the production of three jets and $R_{3 / 2}$ ratios at the LHC at $13 \mathrm{TeV}$. Second, it demonstrates the technical ability to treat the NNLO real radiation contributions for processes with five colored partons at the Born level. The completion of the second order corrections to three jet production is a milestone in perturbative QCD computations since, judging by its infrared structure, it is among the most complicated two-to-three processes at the LHC.

This Letter is organized as follows: in the section "Calculation details" we discuss the technical details of our computation. The section "Results" contains the 
phenomenological results and their analysis. We conclude with a summary and outlook on future applications in the section "Conclusions."

Calculation details.-The computation has been performed within the sector-improved residue subtraction scheme formalism $[28,29]$ which has already been successfully applied to single inclusive jet production [24] and many other hadron collider processes, see Refs. [30-32]. We work in five-flavor massless QCD without the top quark. Tree-level matrix elements have been taken from the AvH library $[33,34]$ while all one-loop matrix elements have been implemented with the OpenLoops library [35]. The double virtual matrix elements are not yet available beyond the leading-color approximation. For this reason we approximate the finite two-loop contribution

$$
\begin{aligned}
\mathcal{R}^{(2)}\left(\mu_{R}^{2}\right) & =2 \Re\left[\mathcal{M}^{\dagger(0)} \mathcal{F}^{(2)}\right]\left(\mu_{R}^{2}\right)+\left|\mathcal{F}^{(1)}\right|^{2}\left(\mu_{R}^{2}\right) \\
& \equiv \mathcal{R}^{(2)}\left(s_{12}\right)+\sum_{i=1}^{4} c_{i} \ln ^{i}\left(\frac{\mu_{R}^{2}}{s_{12}}\right),
\end{aligned}
$$

where $s_{12}=\left(p_{1}+p_{2}\right)^{2}$ the invariant mass of the incoming partons, in the following way:

$$
\mathcal{R}^{(2)}\left(s_{12}\right) \approx \mathcal{R}^{(2) \mathrm{lc}}\left(s_{12}\right),
$$

where $\mathcal{R}^{(2) \mathrm{lc}}\left(s_{12}\right)$ denotes its leading-color approximation. It is taken from the $\mathrm{C}++$ implementation provided in Ref. [27].

Equation (2) above is the only approximation made in the present computation. We have checked that the overall contribution of $\mathcal{R}^{(2) \mathrm{lc}}\left(s_{12}\right)$ is about $\mathcal{O}(2 \%)$ and we expect the missing pure virtual contributions beyond the leadingcolor approximations to be further suppressed.

We consider production of two and three jets at the LHC with a center of mass energy of $13 \mathrm{TeV}$ with jet requirements adapted from experimental phase space definitions like, for example, Ref. [6]. Jets are clustered using the anti$k_{T}$ algorithm [36] with a radius of $R=0.4$ and required to have transverse momentum $p_{T}(j)$ of at least $60 \mathrm{GeV}$ and rapidity $y(j)$ fulfilling $|y(j)|<4$.4. All jets passing this requirement are sorted and labeled according to their $p_{T}$ from largest to smallest. Among those jets we require the two leading jets to fulfill $p_{T}\left(j_{1}\right)+p_{T}\left(j_{2}\right)>250 \mathrm{GeV}$ in order to avoid large higher-order corrections in two-jet production close to the phase space boundary. We denote by $d \sigma_{n}$ the differential cross section for at least $n$ jets fulfilling the above criteria. Its expansion in $\alpha_{S}$ reads

$$
\begin{aligned}
d \sigma_{n} & =d \sigma_{n}^{(0)}+d \sigma_{n}^{(1)}+d \sigma_{n}^{(2)}+\mathcal{O} \alpha_{S}^{n+3} \\
d \sigma_{n}^{\mathrm{LO}} & =d \sigma_{n}^{(0)}, \\
d \sigma_{n}^{\mathrm{NLO}} & =d \sigma_{n}^{(0)}+d \sigma_{n}^{(1)}, \\
d \sigma_{n}^{\mathrm{NNLO}} & =d \sigma_{n}^{(0)}+d \sigma_{n}^{(1)}+d \sigma_{n}^{(2)} .
\end{aligned}
$$

We quantify the size of (N)NLO corrections with the help of the following ratios of differential cross sections:

$$
K^{\mathrm{NNLO}}=\frac{d \sigma^{\mathrm{NNLO}}}{d \sigma^{\mathrm{NLO}}} \quad \text { and } \quad K^{\mathrm{NLO}}=\frac{d \sigma^{\mathrm{NLO}}}{d \sigma^{\mathrm{LO}}} .
$$

The PDF set NNPDF31_nnlo_as_0118 is used for all perturbative orders. The renormalization $\mu_{R}$ and factorization $\mu_{F}$ scales are set equal $\mu_{R}=\mu_{F}=\mu_{0}$. The central scale $\mu_{0}$ is chosen as $\hat{H}_{T} / n$ for $n=1,2$, where

$$
\hat{H}_{T}=\sum_{i \in \text { partons }} p_{T, i} .
$$

The sum in the above equation is over all final state partons, irrespective of the jet requirements. Previous studies of perturbative convergence in jet production support this event-based dynamic scale [37,38]. Unless stated otherwise, uncertainties from missing higher orders in perturbation theory are estimated by variation of $\mu_{F}=\mu_{R}$ by a factor of 2 around the central scale $\mu_{0}$.

The calculation of the three-jet production cross sections is computationally and technically challenging. The main bottlenecks are the double real radiation corrections and the corresponding integrated subtraction terms, due to large numerical cancellation between individual contributions. The numerical evaluation of the complex doublevirtual amplitudes is fast due to the efficient representation presented in [27].

Results.-We begin by discussing typical jet observables at hadron colliders. In Fig. 1 we show differential cross sections for three-jet production with respect to the transverse momentum $p_{T}\left(j_{i}\right)$ of the $i$ th leading jet. In all histograms the outer bins do not include over- or underflow events.

The NNLO $K$ factor of the $p_{T}\left(j_{1}\right)$ distribution is not flat: at small $p_{T}\left(j_{1}\right)$ one observes negative NNLO corrections of about $-10 \%$, while at large $p_{T}\left(j_{1}\right)$ the corrections tend to be small and positive. The change in scale dependence for this observable when going from NLO to NNLO is also dependent on $p_{T}\left(j_{1}\right)$. One observes a rather significant reduction at large $p_{T}\left(j_{1}\right)$ (from about $7 \%$ at NLO to about $2 \%$ at NNLO) while at small $p_{T}\left(j_{1}\right)$, where the $K$ factor is largest, the scale dependence slightly increases (from about $4 \%$ at NLO to about 5\% at NNLO). Interestingly, the scale dependence at NLO and NNLO behaves rather differently: at NLO it steadily increases with $p_{T}\left(j_{1}\right)$ while at NNLO it decreases with $p_{T}\left(j_{1}\right)$. Throughout this work we define the scale dependence as one half of the width of the scale uncertainty band. This is relevant for cases where the scale variation is asymmetric, as for example is the case of $p_{T}\left(j_{1}\right)$ at NLO.

The $p_{T}\left(j_{2}\right)$ distribution has a similar pattern of NNLO corrections: relative to NLO they are negative, about $-20 \%$ at low $p_{T}\left(j_{2}\right)$, and steadily increase towards larger $p_{T}\left(j_{2}\right)$ 


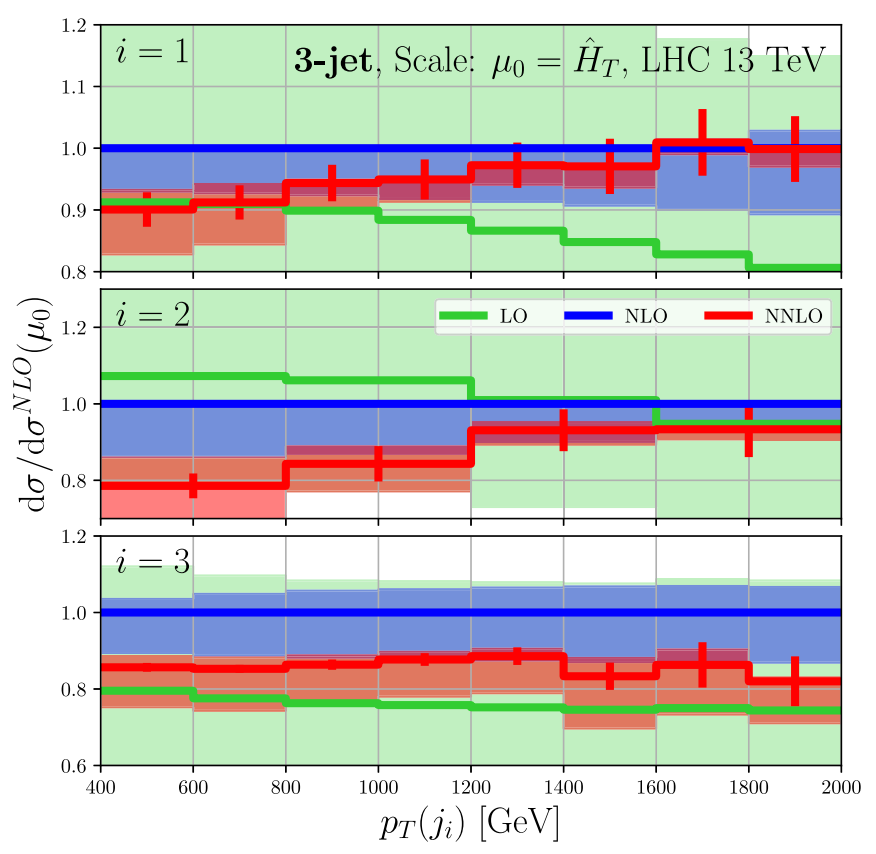

FIG. 1. The three panels show the $i$ th leading jet transverse momentum $p_{T}\left(j_{i}\right)$ for $i=1,2,3$ for the production of (at least) three jets. LO (green), NLO (blue), and NNLO (red) are shown for the central scale (solid line). Three-point scale variation is shown as a colored band. The grey band corresponds to the uncertainty from Monte Carlo integration.

values. At both NLO and NNLO the scale dependence of $p_{T}\left(j_{2}\right)$ is similar to that of $p_{T}\left(j_{1}\right)$. On the technical side, the convergence of the numerical integration for the $p_{T}\left(j_{2}\right)$ spectrum has been significantly slower than for the other $p_{T}$ observables, which results in increased Monte Carlo uncertainty. To compensate for this, a larger bin size has been used for $p_{T}\left(j_{2}\right)$. Independently of its slower numerical convergence, the $p_{T}\left(j_{2}\right)$ spectrum shows good perturbative convergence. Such a behavior is in contrast to the two-jet case where the subleading $p_{T}$ spectrum is known to get large perturbative corrections due to the strict back-to-back tree-level kinematics [38].

The $p_{T}\left(j_{3}\right)$ distribution is well behaved: it has a flat $K$ factor and fairly symmetric uncertainty band at both NLO and NNLO. The scale variation is almost independent of $p_{T}\left(j_{3}\right)$, about the $5 \%$ at NNLO, which is only slightly smaller than in the NLO case.

The fact that this observable shows such good convergence and perturbative stability is somewhat remarkable. Naively, one may suspect that the scale used here may not perform very well for this distribution because the scale is based on the kinematics of the full event which, in turn, is dominated by the leading jet(s). Since this finding may be of relevance for the extraction of the strong coupling constant from three-jet events, it may be worth investigating this behavior in more detail. This is outside the scope of the present work.

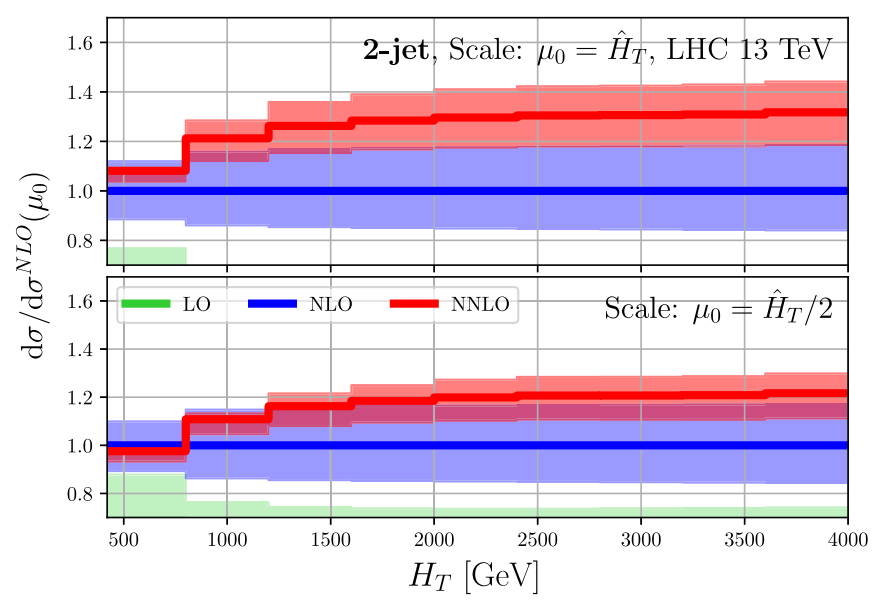

FIG. 2. The observable $H_{T}$ in two-jet production for two different central scale choices. Scale variation corresponds to three-point variation. The colors are the same as in Fig. 1.

Next we discuss the observable $H_{T}$, defined as

$$
H_{T}=\sum_{i \in \text { jets }} p_{T}\left(j_{i}\right)
$$

where the sum is over all jets that pass the jet requirements. We show this observable in Fig. 2 for the two-jet process and in Fig. 3 for the three-jet process. Both figures are subdivided in two panels showing the same observable but for a different central scale choice: the upper panels for $\mu_{0}=\hat{H}_{T}$ and the lower panels for $\mu_{0}=\hat{H}_{T} / 2$. Turning to the two-jet case we see that both the perturbative convergence and the scale dependence improve if the central scale choice is lowered. For $\mu_{0}=\hat{H}_{T}$ the inclusion of the NNLO QCD corrections does not reduce significantly the scale dependence with respect to NLO and both bands barely overlap. However, $K^{\mathrm{NNLO}} \approx 1.2$ is much smaller than $K^{\mathrm{NLO}} \approx 2$ indicating the stabilization of higher-order corrections beyond NNLO. For the production of three jets

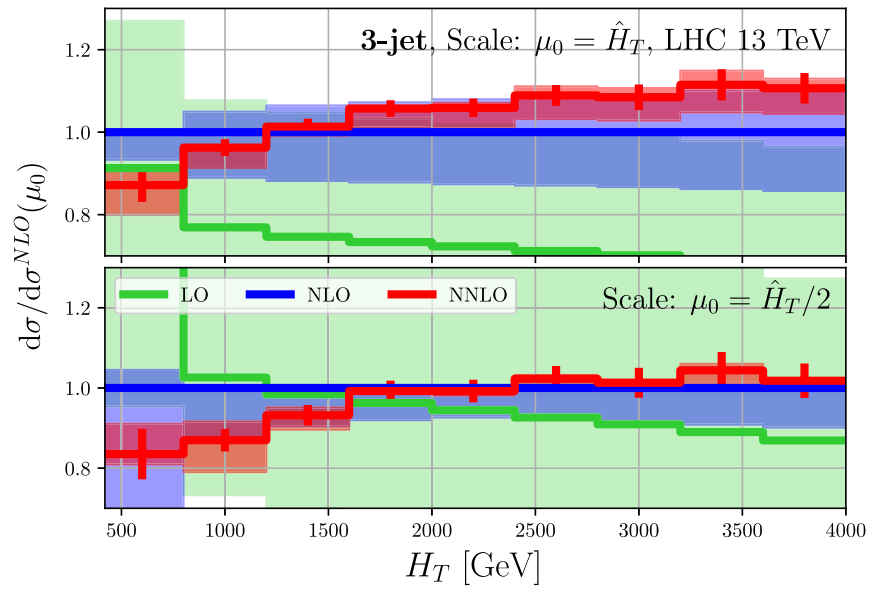

FIG. 3. As in Fig. 2 but for three-jet production. 
we find that the two central scale choices $\mu_{0}=\hat{H}_{T}$ and $\mu_{0}=\hat{H}_{T} / 2$ produce comparable results, albeit $\mu_{0}=\hat{H}_{T} / 2$ has smaller scale variation. The scale dependence is small compared to the two-jet case and $K^{\mathrm{NNLO}}$ is closer to one. These findings indicate that a central scale of $\mu_{0}=\hat{H} / 2$ leads to slightly better perturbative convergence and, thus, better approximates the actual energy scale relevant for this observable. We have checked that even lower scales, like $\mu_{0}=\hat{H}_{T} / 4$, spoil perturbative convergence.

As a first application of the NNLO-accurate three-jet rates computed in this work we consider ratios between three-jet and two-jet rates. The ratios are defined as

$$
R_{3 / 2}\left(X, \mu_{R}, \mu_{F}\right)=\frac{d \sigma_{3}\left(\mu_{R}, \mu_{F}\right) / d X}{d \sigma_{2}\left(\mu_{R}, \mu_{F}\right) / d X},
$$

where $X$ is some observable of interest. The $(\mathrm{N}) \mathrm{NLO}$ ratio is defined in such a way that the numerator and denominator on the right-hand side are evaluated at the matching order. The scale dependence of the differential cross sections is shown explicitly to emphasize that the scale choices in the numerator and denominator are correlated.

In the upper two panels of Fig. 4 we show the ratio $R_{3 / 2}\left(p_{T}\left(j_{1}\right)\right)$. The ratio changes drastically when going from LO to NLO mostly due to the change in the two-jet cross section. The NNLO correction stabilizes the ratio and leads to a very small scale dependence. The $K^{\mathrm{NNLO}}$ factor slightly decreases for large momenta, however, it is always fully contained within the NLO scale band. An important observation is that the NNLO scale band is very small in comparison to NLO, reducing it from about $10 \%$ down to $3 \%$.

Next we consider the lower two panels in Fig. 4, where we show the ratio $R_{3 / 2}\left(H_{T}\right)$ for a central scale $\mu_{0}=H_{T} / 2$. This observable behaves similarly to $R_{3 / 2}\left[p_{T}\left(j_{1}\right)\right]$ albeit with a slightly larger scale dependence. The reduction in the scale uncertainty when going from NLO to NNLO is of particular importance since this observable is used experimentally for measurements of $\alpha_{S}$ [5]. The leading source of perturbative uncertainty in this data-theory comparison is the scale dependence. The PDF dependence, which is not computed in this work, is expected to be reduced in the ratio.

Jet rates are typically measured in slices of jet rapidity. To demonstrate how our calculation performs in this situation, we divide the phase space in slices of the rapidity difference between the two leading jets

$$
y^{*}=\left|y\left(j_{1}\right)-y\left(j_{2}\right)\right| / 2,
$$

and define the ratio of the two- and three-jet rates as

$$
R_{3 / 2}\left(H_{T}, y^{*}\right)=\frac{d^{2} \sigma_{3} / d H_{T} / d y^{*}}{d^{2} \sigma_{2} / d H_{T} / d y^{*}} .
$$
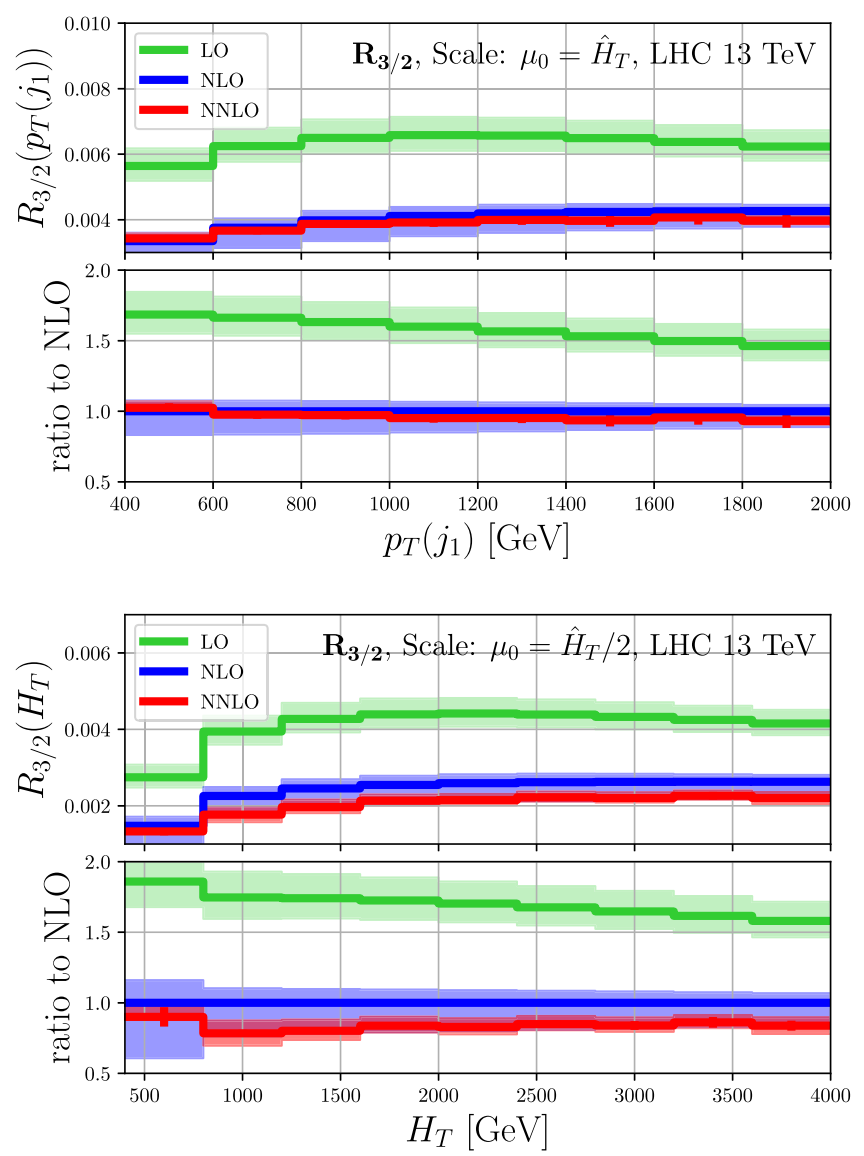

FIG. 4. The top two panels show $R_{3 / 2}\left(p_{T}\left(j_{1}\right)\right.$ ) (in absolute and as ratio to NLO) and the bottom two panels $R_{3 / 2}\left(H_{T}\right)$. The colors are the same as in Fig. 1.

The NNLO prediction for this cross section ratio can be found in Fig. 5. The prediction is shown relative to the NLO one. The NNLO correction is negative across the full kinematic range and, overall, behaves very similarly to the one for the rapidity-inclusive ratio $R_{3 / 2}\left(H_{T}\right)$. This remains

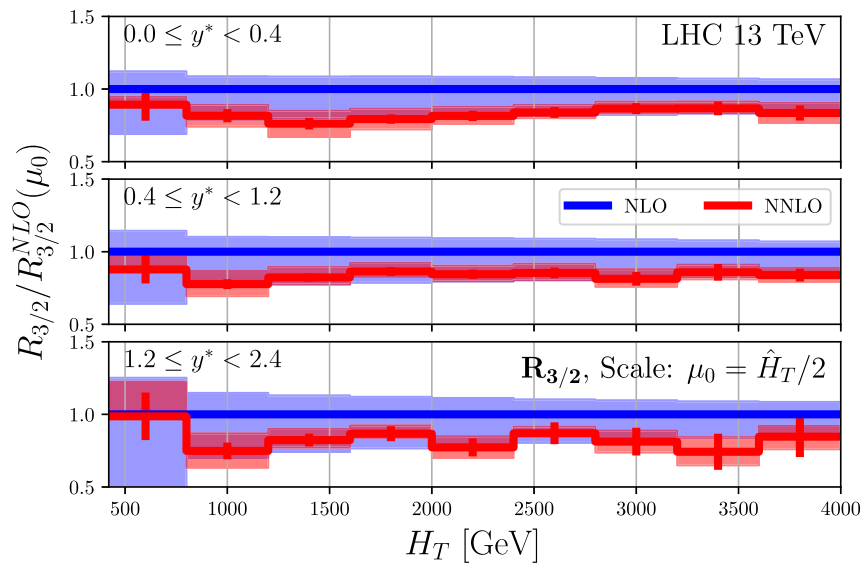

FIG. 5. The three panels show $R_{3 / 2}\left(H_{T}, y^{*}\right)$, in each panel a different slice in $y^{*}$ as ratio to NLO. The colors are the same as in Fig. 1. 
the case as $y^{*}$ increases, at least in the range of rapidities considered here.

Conclusions. - In this work we present for the first time NNLO-accurate predictions for three-jet rates at the LHC. We compute differential distributions for typical jet observables like $H_{T}$ and the transverse momentum of the $i$ th leading jet, $i=1,2,3$, as well as differential threeto-two jet ratios. Scale dependence is the main source of theoretical uncertainty for this process at NLO, and it gets significantly reduced after the inclusion of the NNLO QCD corrections. Notably, the three-to-two jet ratios stabilize once the second-order QCD corrections are accounted for.

A central goal of the present work is to demonstrate the feasibility of three-jet hadron collider computations with NNLO precision. With this proof-of-principle goal attained, one can now turn one's attention to the broad landscape of phenomenological applications for three-jet production at the LHC. Examples include studies of event shapes $[6,39,40]$, determination of the running of the strong coupling constant $\alpha_{s}$ through TeV scales, and resolving the question of scale setting in multijet production. Another major benefit from having NNLO-accurate predictions is the reliability of the theory uncertainty estimates.

On the technical side, the enormous computational cost of the present calculation $\left(\sim 10^{6} \mathrm{CPUh}\right)$ makes it clear that further refinements in the handling of real radiation contributions to NNLO calculations are desirable.

We would like to thank Manuel Alvarez, Javier Llorente, and Jennifer Roloff for helpful discussions about ATLAS jet measurements. The work of M. C. was supported by the Deutsche Forschungsgemeinschaft under Grant No. 396021762-TRR 257. The research of A. M. and R.P. has received funding from the European Research Council (ERC) under the European Union's Horizon 2020 Research and Innovation Programme (Grant Agreement No. 683211). A. M. was also supported by the UK STFC grants ST/L002760/1 and ST/K004883/1. A. M. acknowledges the use of the Distributed Research utilising Advanced Computing (DiRAC) Cumulus HPC facility under Grant No. PPSP226. Simulations were performed with computing resources granted by RWTH Aachen University under project rwth0414.

[1] G. Aad et al. (ATLAS Collaboration), Eur. Phys. J. C 71, 1763 (2011).

[2] G. Aad et al. (ATLAS Collaboration), Eur. Phys. J. C 75, 228 (2015).

[3] V. Khachatryan et al. (CMS Collaboration), Eur. Phys. J. C 75, 186 (2015).

[4] G. Aad et al. (ATLAS Collaboration), Phys. Lett. B 750, 427 (2015).

[5] M. Aaboud et al. (ATLAS Collaboration), Phys. Rev. D 98, 092004 (2018).
[6] G. Aad et al. (ATLAS Collaboration), J. High Energy Phys. 01 (2021) 188.

[7] S. Chatrchyan et al. (CMS Collaboration), Eur. Phys. J. C 73, 2604 (2013).

[8] S. D. Ellis, Z. Kunszt, and D. E. Soper, Phys. Rev. Lett. 69, 1496 (1992).

[9] W. T. Giele, E. W. N. Glover, and D. A. Kosower, Nucl. Phys. B403, 633 (1993).

[10] Z. Nagy, Phys. Rev. Lett. 88, 122003 (2002).

[11] Z. Nagy, Phys. Rev. D 68, 094002 (2003).

[12] Z. Bern, G. Diana, L. J. Dixon, F. F. Cordero, S. Hoeche, D. A. Kosower, H. Ita, D. Maitre, and K. Ozeren, Phys. Rev. Lett. 109, 042001 (2012).

[13] S. Badger, B. Biedermann, P. Uwer, and V. Yundin, Phys. Rev. D 89, 034019 (2014).

[14] S. Dittmaier, A. Huss, and C. Speckner, J. High Energy Phys. 11 (2012) 095.

[15] R. Frederix, S. Frixione, V. Hirschi, D. Pagani, H. S. Shao, and M. Zaro, J. High Energy Phys. 04 (2017) 076.

[16] M. Reyer, M. Schönherr, and S. Schumann, Eur. Phys. J. C 79, 321 (2019).

[17] S. Alioli, K. Hamilton, P. Nason, C. Oleari, and E. Re, J. High Energy Phys. 04 (2011) 081.

[18] S. Hoche and M. Schonherr, Phys. Rev. D 86, 094042 (2012).

[19] T. Gleisberg, S. Hoeche, F. Krauss, M. Schonherr, S. Schumann, F. Siegert, and J. Winter, J. High Energy Phys. 02 (2009) 007.

[20] J. Alwall, R. Frederix, S. Frixione, V. Hirschi, F. Maltoni, O. Mattelaer, H. S. Shao, T. Stelzer, P. Torrielli, and M. Zaro, J. High Energy Phys. 07 (2014) 079.

[21] R. Frederix, S. Frixione, V. Hirschi, D. Pagani, H. S. Shao, and M. Zaro, J. High Energy Phys. 07 (2018) 185.

[22] J. Currie, E. W. N. Glover, and J. Pires, Phys. Rev. Lett. 118, 072002 (2017).

[23] J. Currie, A. Gehrmann-De Ridder, T. Gehrmann, E. W. N. Glover, A. Huss, and J. Pires, Phys. Rev. Lett. 119, 152001 (2017).

[24] M. Czakon, A. van Hameren, A. Mitov, and R. Poncelet, J. High Energy Phys. 10 (2019) 262.

[25] R. Abdul Khalek, S. Forte, T. Gehrmann, A. Gehrmann-De Ridder, T. Giani, N. Glover, A. Huss, E. R. Nocera, J. Pires, J. Rojo et al., Eur. Phys. J. C 80, 797 (2020).

[26] D. Chicherin and V. Sotnikov, J. High Energy Phys. 12 (2020) 167.

[27] S. Abreu, F. Febres Cordero, H. Ita, B. Page, and V. Sotnikov, J. High Energy Phys. 07 (2021) 095.

[28] M. Czakon, Phys. Lett. B 693, 259 (2010).

[29] M. Czakon and D. Heymes, Nucl. Phys. B890, 152 (2014).

[30] M. Czakon, D. Heymes, and A. Mitov, Phys. Rev. Lett. 116, 082003 (2016).

[31] H. A. Chawdhry, M. Czakon, A. Mitov, and R. Poncelet, J. High Energy Phys. 09 (2021) 093.

[32] H. A. Chawdhry, M. L. Czakon, A. Mitov, and R. Poncelet, J. High Energy Phys. 02 (2020) 057.

[33] https://bitbucket.org/hameren/avhlib.

[34] M. Bury and A. van Hameren, Comput. Phys. Commun. 196, 592 (2015). 
[35] F. Buccioni, J. N. Lang, J. M. Lindert, P. Maierhöfer, S. Pozzorini, H. Zhang, and M. F. Zoller, Eur. Phys. J. C 79, 866 (2019).

[36] M. Cacciari, G. P. Salam, and G. Soyez, J. High Energy Phys. 04 (2008) 063.

[37] J. Bellm, A. Buckley, X. Chen, A. Gehrmann-De Ridder, T. Gehrmann, N. Glover, A. Huss, J. Pires, S. Höche, J. Huston et al., Eur. Phys. J. C 80, 93 (2020).
[38] J. Currie, A. Gehrmann-De Ridder, T. Gehrmann, E. W. N. Glover, A. Huss, and J. Pires, J. High Energy Phys. 10 (2018) 155.

[39] A. Banfi, G. P. Salam, and G. Zanderighi, J. High Energy Phys. 08 (2004) 062.

[40] A. Banfi, G. P. Salam, and G. Zanderighi, J. High Energy Phys. 06 (2010) 038. 